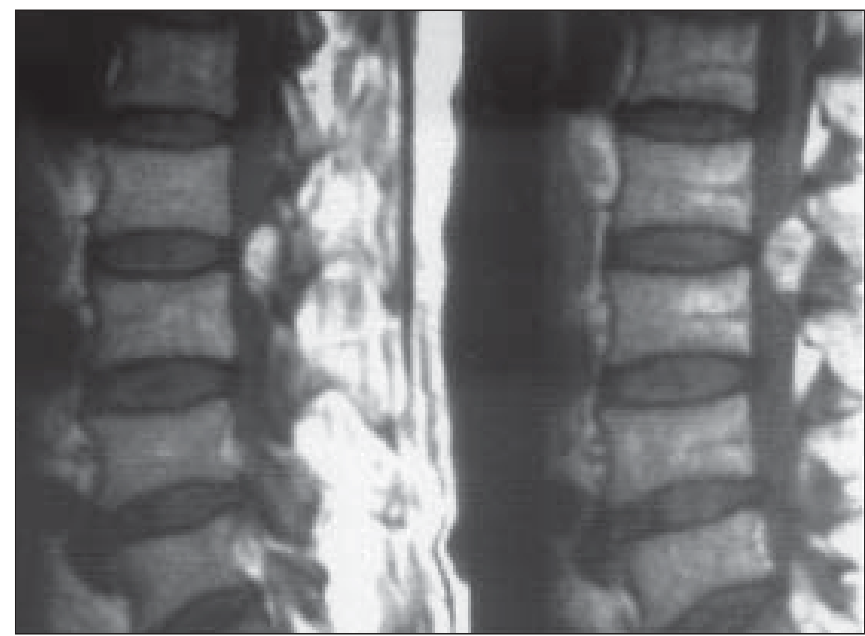

Figure 1: Noncontrast sagittal lumbar T1W magnetic resonance imaging (MRI) demonstrated a localized high-intensity fusiform mass at the L2-3 disc level, located posteriorly

\section{Lumbar epidural hematoma due to lumbar acupunctures}

Sir,

Although complications associated with acupuncture have been reported,,$^{[1,2]}$ they are often transient and mild. Serious complications following acupuncture are rare ${ }^{[1]}$ In this report, we present a case of localized lumbar epidural hematoma following acupunctures.

A 74-year-old man presented with complaints of progressive weakness of the left leg and radicular pain in the left thigh for 2 days following the last session of acupunctures in the lumbar region for his back pain. He was hospitalized about 4 weeks later. There was no other systemic complaint. There was no history suggestive of any bleeding disorder. On physical examination, it was detected that he had left-thigh weakness $(4 / 5)$ and absence of left-knee jerk. He did not have neck rigidity; no obvious sensory deficit was revealed during the physical examination.

Noncontrast T1W magnetic resonance imaging (MRI) demonstrated a localized high-intensity fusiform mass at the L23 dise level, located posteriorly [Figure 1 and 2]. The results of laboratory coagulation tests were normal.

An appropriate laminectomy was performed. An organized encapsulated hematoma was encountered; the capsule was attached to the dura. There was no dural tear or any clear sign of previous punctures. The results of the pathologic examination of the specimens (the capsule and its content) were

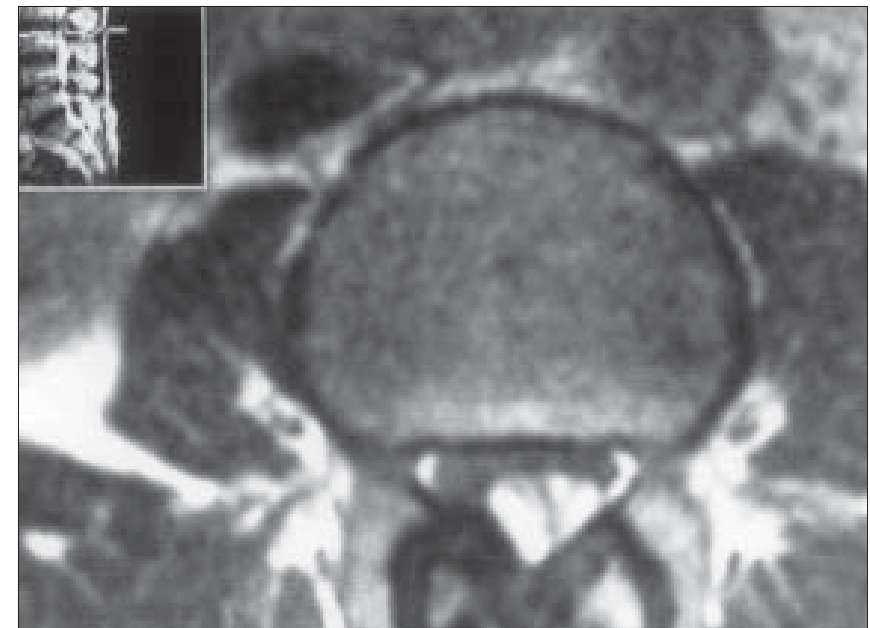

Figure 2: In the axial T1W magnetic resonance imaging, the mass is asymmetric, slightly more voluminous on the left

fibrosis and hematoma. One week after the operation, the patient recovered completely from the neurological deficit and was relieved of the radicular pain.

Although serious complications have been reported with acupuncture, they are rare. Yamashita suggests that most serious adverse events are actually cases of negligence. ${ }^{[2]}$ We found two cases of spinal epidural hematoma associated with acupuncture in the Medline. ${ }^{[3,4]}$ Chen $^{[3]}$ reported a 48-year-old female with meningeal irritation within 1 week after the acupunctures, leading to a L1/2 subacute epidural hematoma and meningitis. The patient was managed conservatively. Keane ${ }^{[t]}$ reported a thoracic spinal epidural hemorrhage with subarachnoid hemorrhage following acupunctures.

This case draws attention to the rare complication of the lumbar epidural hematoma following acupunctures. The history and the localized nature of the hematoma should raise suspicion. Depending on the clinical course, either a conserva- 
tive observation or surgical evacuation of the clot can be the treatment options.

Behzad Eftekhar, Ebrahim Ketabchi, Mohammad Ghodsi, Babak Esmaeeli

Department of Neurosurgery, Sina Hospital, Tehran University, Iran. E-mail: eftekhar@sina.tums.ac.ir

\section{References}

1. Yamashita H, Tsukayama H, Hori N, Kimura T, Tanno Y. Incidence of adverse reactions associated with acupuncture. J Altern Complement Med 2000;6:34550 .

2. Yamashita H, Tsukayama H, Tanno Y, Nishijo K. Adverse events in acupuncture and moxibustion treatment: A six-year survey at a national clinic in Japan. J Altern Complement Med 19995:229-36.

3. Chen CY, Liu GC, Sheu RS, Huang CL. Bacterial meningitis and lumbar epidural hematoma due to lumbar acupunctures: A case report. Kaohsiung J Med Sci 1997;13:328-31.

4. Keane JR, Ahmadi J, Gruen P. Spinal epidural hematoma with subarachnoid hemorrhage caused by acupuncture. A.JNR Am J Neuroradiol 1993;14:365-6.

Accepted on 19-01-2005 